\title{
Attentional Biases in Processing Emotional Facial Expressions: Effects of State Anxiety, Trait Anxiety and Awareness
}

\author{
Mark S. Edwards ${ }^{1,2}$ \\ ${ }^{1}$ School of Psychology, University of Queensland, Brisbane, Australia \\ ${ }^{2}$ School of Psychology, Bond University, Gold Coast, Australia \\ Correspondence: Mark S. Edwards, School of Psychology, Bond University, Gold Coast, Q 4229, Australia. Tel: \\ 61-7-5595-2673. E-mail: medwards@bond.edu.au
}

Received: July 27, 2014

doi:10.5539/ijps.v6n4p41
Accepted: August 19, $2014 \quad$ Online Published: November 40, 2014

URL: http://dx.doi.org/10.5539/ijps.v6n4p41

\begin{abstract}
The effect of state anxiety and trait anxiety on selective attention for threatening, neutral and happy schematic faces was investigated. A student sample was assigned to high trait anxious (HTA) or low trait anxious (LTA) groups using questionnaire scores and state anxiety was manipulated through the threat of electric shock. Stimulus materials were presented both outside (using a backward masking procedure) and within awareness. A novel version of a probe classification task was developed to assess performance when distracting information and the central task were spatially separated. For the masked trials, the LTA group were slower to identify the status of the probe on happy face trials relative to threat face trials in the shock safe condition, and slower on threat face trials relative to happy face trials in the shock threat condition. For the HTA group, performance on the probe status task was unaffected by shock condition status or item valence for the masked exposures. During the unmasked exposure trials neither trait anxiety status, item valence nor shock condition affected response latencies to the probes. Results are interpreted with respect to contemporary models of attention and anxiety.
\end{abstract}

Keywords: attentional bias, anxiety, backward masking, threat faces

\section{Introduction}

Widely cited theories of cognition and emotion support the view that anxious individuals assign attentional priority to threat related information (e.g., Bower, 1981; Mogg \& Bradley 1998; Williams, Watts, MacLeod \& Mathews, 1988, 1997). These theories suggest that threat processing biases operate automatically in that they occur without intention and awareness, for clinically and non-clinically anxious individuals alike. These viewpoints, however, do not agree with respect to the relative effects of state and trait anxiety on attentional processes. Bower's semantic network model, for example, predicts a mood congruent bias in favour of emotionally congruent stimuli, such that anxious individuals selectively process information relevant to their current mood state. Williams et al. and Mogg and Bradley, however, suggest that state and trait anxiety combine to moderate selective attention such that threat biases are restricted to high trait anxious (HTA) individuals who experience elevated levels of state anxiety. Although similar in their predictions for HTA individuals, the Williams et al. and Mogg and Bradley models make separate assumptions regarding the attentional deployment of low trait anxious (LTA) individuals. Whereas Williams et al. suggest that elevated state anxiety and/or threat value are associated with avoidance of threat in LTA participants, Mogg and Bradley argue that stimuli appraised as carrying a high threat value will recruit attention independently of trait anxiety status. Threat processing biases have been implicated as causal and maintaining factors in anxiety (e.g., Williams et al., 1988, 1997) and therefore research into factors that elicit them is important.

Most of the evidence supporting these theoretical positions has been derived from research employing well established cognitive experimental paradigms such as the emotional Stroop and dot-probe tasks. In the emotional Stroop, threat-related (e.g., infection, illness) and neutral (e.g., spoon, curtain) words are presented to high anxious and non-anxious individuals in letter strings of a particular colour. The participants' task is to ignore the semantic content and name the colour of the item as quickly as possible. An attentional bias is inferred by differential colour naming latencies for threatening words relative to neutral words, presumably because the semantic nature of the item disrupts colour naming performance. Given that participants are instructed to ignore the meaning of the item and to name its colour as quickly as possible, the task seems well-suited to assess the 
idea that threat bias effects operate without volition. Indeed, there is now considerable evidence derived from this methodology demonstrating threat related attentional biases in clinical populations (e.g., Amir, Freshman, \& Foa, 2002; Bradley, Mogg, Millar, \& White, 1995; Cohen, Lachenmeyer, \& Springer, 2003; Harvey, Bryant, \& Rapee, 1996; Lundh, Wikström, Westerlund, \& Öst, 1999; McNally, Riemann, \& Kim, 1990), and in high trait anxious individuals reporting elevated state anxiety (e.g., Edwards, Burt, \& Lipp, 2006; Edwards, Burt, \& Lipp, 2010a; Miller \& Patrick, 2000). There is also substantial data from the emotional Stroop under backward masking conditions to demonstrate that these effects operate without awareness in clinically anxious (e.g. Bradley et al., 1995; Foa, Feske, Murdock, Kozak, \& McCarthy, 1991; Harvey et al., 1996; Lundh et al., 1999) and HTA participants experiencing elevated state anxiety (e.g. Edwards et al., 2006; MacLeod \& Rutherford, 1992).

A considerable volume of research has also employed dot-probe detection or classification tasks to investigate threat biases in anxiety. In a prototypical version of this task, a series of word pairs (one threat word and one neutral word) are presented on a computer screen (e.g., for $500 \mathrm{~ms}$ ) with one member of the pair in the left portion of the display and the other member in the right portion of the display. Typically, threat and neutral words appear in either location with equal frequency. Immediately following the offset of the stimulus pair, a visual probe is presented in the location formerly occupied by one of the words and participants are required to respond as rapidly as possible to the location or shape of the probe. An attentional bias is inferred on the basis of faster response latencies for probes replacing threat words relative to neutral words because responses will be facilitated by attention already being allocated to a particular location. Substantial evidence has confirmed faster responses to probes replacing threat stimuli compared to neutral stimuli across a range of clinical anxiety pathologies (e.g., Asmundson \& Stein, 1994; MacLeod, Mathews, \& Tata, 1986; Mogg, Mathews, \& Eysenck, 1992; Tata, Leibowitz, Prunty, Cameron, \& Pickering, 1996) and in highly anxious non-clinical participants (e.g., Broadbent \& Broadbent, 1988; MacLeod \& Mathews, 1988; Mogg, Mathews, Bird, \& Macgregor-Morris, 1990; Mogg, Bradley, \& Hallowell, 1994; Mogg, McNamara et al., 2000). Studies employing masked dot-probe procedures have also reported facilitated probe latencies for probes replacing threat words, relative to control words in clinically anxious (e.g., Mogg, Bradley, \& Williams, 1995; Mogg et al., 1992) and in non-clinical, high anxious participants (e.g., Hunt, Keogh, \& French, 2006).

An important constraint with the use of verbal stimuli in previous Stroop and probe studies is that words are merely symbolic representations for actual threat, and therefore are likely to be relatively limited in their threat value compared to more ecologically valid threat (cf. Mogg \& Bradley), such as pictures of angry or threatening human faces, which represent a special stimulus for humans given their biological and social significance (e.g., Koster, Verschuere, Burssens, Custers, \& Crobmez, 2007). There are now some data using dot-probe methodology and pictures of human faces as the stimuli that have demonstrated faster probe detection/classification latencies for threatening faces relative to non-threatening faces in studies using subliminal (e.g., Mogg \& Bradley, 1999) and supraliminal exposure thresholds (e.g., Bradley, Mogg, Falla, \& Hamilton, 1998; Wilson \& MacLeod, 2003). However, reports of attentional biases for threatening faces using emotional Stroop procedures have been more difficult to capture (e.g., van Honk, Tuiten, de Haan, van den Hout, \& Stam, 2001).

The use of pictures of human faces as stimuli represents a significant step forward in establishing the ecological validity of the threat bias effect in anxiety. Öhman, Lundqvist and Esteves (2001), however, noted that there are at least two problems associated with utilising photographs of human faces as stimuli. First, participants may find some difficulty distinguishing the threat value of photographed threatening and neutral faces, such that a neutral face devoid of any positive emotion might be interpreted as aggressive. Second, whereas most individuals are able to produce a relatively convincing happy smile on command, it is more difficult to produce a credible threatening face at will (cf. Öhman et al.). Nevertheless, the problems associated with using photographic pictures of faces can be overcome if schematic pictures of human faces are employed because the physical features of the faces can be tightly controlled (cf. Öhman et al.).

Despite considerable support for the notion that anxiety is associated with a selective attentional bias for verbal and pictorial threat, there are a number of interpretational constraints inherent with dot-probe and emotional Stroop procedures that do not permit clear interpretation of the models under investigation (i.e., Bower, 1981; Mogg \& Bradley 1998; Williams et al., 1988, 1997). For example, dot-probe tasks employ a time lag between the presentation of the stimulus pairs and onset of the probe and it therefore seems plausible that on unmasked trials in particular, participants might choose to direct their attention towards items of a particular emotional tone before the probe is presented (cf. Edwards, Burt, \& Lipp 2010b). The lack of competition for attentional resources does not therefore permit an evaluation of the "without volition" component of the automaticity 
hypothesis which is central to the models under investigation. In addition, there is evidence to indicate that the probe task is not an internally consistent or stable measure of attentional bias in non-clinical samples (Schmukle, 2005). The emotional Stroop has also come under criticism because the cognitive processes underlying the effect are not well understood (see e.g., Thorpe \& Salkovskis, 1997) and it is unable to evaluate the allocation of spatial attention (MacLeod et al., 1986).

A further concern with both the emotional Stroop and dot-probe tasks rests with the fact that the stimuli are presented in task-relevant locations. In the probe detection task, the probe typically replaces the location of one of the items, and therefore the location of both the probe and the item is task-relevant; with the Stroop, the to-be-attended information (item colour) and the to-be-ignored information (semantic content) are amalgamated aspects of a single stimulus (cf. Fox, 1996). In naturalistic settings it may be unlikely that the focus of attention would be in the exact location that threat appears; an effective threat detection system should be efficient in allocating attention to sources of threat in locations not central to on-going activities. A more appropriate test of the idea that anxiety is associated with an attentional bias for threat would be to present threat information in a location other than that which is focal to the central task.

To overcome the limitations associated with the dot-probe and emotional Stroop tasks a novel interference task was developed. If it is accepted that participants follow the instruction to ignore information not central to the primary task, interference methodologies appear well suited to assess the volitional component of selective attention because they require competition for attentional resources. The task was designed such that threat, neutral and happy faces were presented in locations not focal to the central task. To increase the likelihood that attention was focussed at the centre of the computer screen rather than in task relevant locations, participants performed a digit classification task prior to the main task on each trial (see Mogg \& Bradley, 1998 for further discussion). Once the computer detected the participants' response to the digit, the screen was blanked and a small probe (either a circle or square) was presented in the periphery of the computer screen, either to the left or to the right of the location formerly occupied by the digit. At the onset of the probe, one of three schematic faces (threatening, neutral or happy) was presented concurrently above and below the location formerly occupied by the digit. Happy faces were included to investigate whether biases for threat reflect a general emotionality effect rather than threat-based effects (cf. Rutherford, MacLeod, \& Campbell, 2004). The participants' task was to verbally classify the probe as either a circle or square as quickly and accurately as possible while ignoring the facial information. To assess the idea that attention to threat operates without awareness, half the trials were backward masked. Slowed response times to probes presented on trials in which items of a particular valence were present would indicate the extent to which participants could avoid attending to distracting faces. In order to evaluate the separate effects of trait anxiety and state anxiety on selective attention, individuals were assigned to the HTA and LTA groups using questionnaire scores responses and state anxiety was manipulated using the threat of electric shock.

In line with the models of Bower (1981), Williams et al. (1988, 1997) and Mogg and Bradley (1988) the following predictions were made. According to Bower, individuals performing under the threat of shock would be slower to identify the status of probes on masked and unmasked threat face trials relative to non-threatening face trials. According to Williams et al., HTA individuals performing under the threat of shock should be significantly slower to identify the status of the probe on masked and unmasked threat faces trials relative to non-threatening face trials, whereas LTA participants should be significantly faster at identifying the status of the probe on threat face trials relative to non-threat trials. According to Mogg and Bradley, both HTA and LTA individuals performing under the threat of shock would be slower to classify the probe on threat face trials relative to the non-threatening face trials in both the masked and unmasked exposure modes.

\section{Method}

\subsection{Participants}

Ninety-six undergraduate students were recruited and provided informed consent. An invitation to participate was offered to those who reported English as their native language, normal colour vision, and normal (or corrected to normal) vision. Only individuals who scored 16 points or below on the Beck Depression Inventory-Revised (BDI-IA; Beck \& Steer, 1993) were retained because previous research had suggested that masked threat effects in anxiety might be attenuated by higher levels of depression (e.g., Bradley et al., 1995). Because social desirability has shown to be associated with a unique pattern of attentional deployment (see Weinberger, Schwartz, \& Davidson, 1979), participation was restricted to those with a score of five or lower socially desirable responses on the Marlowe-Crowne Social Desirability Scale-Form XI (MCSDS; Crowne \& Marlowe, 1960; Strahan \& Gerbasi, 1972). At the request of the university ethics committee, participants whose 
scores were above 65 points on either form of the State Trait Anxiety Inventory (STAI; Spielberger, Gorsuch, Lushene, Vagg, \& Jacobs, 1983) were excluded to protect them from exposure to anxiety invoking stimuli (i.e., threatening stimuli; electric shock). Of those initially recruited, 1 was rejected because her score fell above the inclusion criterion for the STAI-T, 6 were discarded due to high depression, 11 due to high social desirability, and 1 due to both high social desirability and high depression. Of the remaining individuals who met the initial screening criteria, 1 withdrew because she reported having a headache, 1 withdrew when she was reminded that an electric stimulus was an experimental manipulation, 1 due to equipment failure, and 1 because of experimenter error. In addition to these exclusions, the data from a further 9 participants were omitted from the final analyses due to above criterion performance on the final awareness checking trials. All 96 individuals were awarded experimental credit for their participation.

The final sample consisted of 64 participants (32 HTA; 32 LTA) aged between 17 and 39 years $(M=19.95$ years). Seven were male and 57 were female. In line with previous research (e.g., Edwards et al., 2010a, 2010b; MacLeod \& Rutherford, 1992) and normative median for the measure, participants who scored 37 and above on the STAI-T were assigned to the HTA group whereas those who scored lower than 37 were assigned to the LTA group. The HTA and LTA groups did not differ on age, $t<1$, and sex was proportionately represented across the groups (HTA $=3$ males \& 29 females; LTA $=4$ males $\& 28$ females).

\subsection{Materials}

Pictorial stimuli. The schematic representations of happy, neutral, and threatening faces were adapted from those employed in previous research using a visual search paradigm (Öhman et al., 2001; see also Lundqvist, Esteves, \& Öhman, 1999) and are shown in Figure 1. Six scrambled faces were developed for use in the practice and final awareness checking trials, and these are shown in Figure 2. The scrambled faces were produced by horizontally interchanging the facial features of the three face types. In one arrangement the nose replaced the position of the eyes, the mouth replaced the position of the nose, and the eyes and eyebrows replaced the position of the mouth. In the other arrangement the mouth replaced the location of the eyes, the eyes and eyebrows replaced the location of the nose, and the nose replaced the location of the mouth. This procedure ensured that the scrambled faces were matched for line thickness, line length and luminance with the face stimuli used in the main task. Four pattern masks were produced by randomly rearranging the features of the face stimuli. To make it difficult for the features of the targets to leak through the masks, the masks were more visually complex than the face and scrambled face stimuli. There was a proportionate representation of the stimulus features from the happy, neutral and threatening faces in each mask. The pattern masks are shown below in Figure 3. All stimuli (i.e., faces, scrambled faces, and pattern masks) were presented in the centre of the monitor in dimensions approximately 60 $\mathrm{mm}$ high X $50 \mathrm{~mm}$ wide in black colouring. The centre-to-centre separation of the distracter faces was approximately $70 \mathrm{~mm}$.
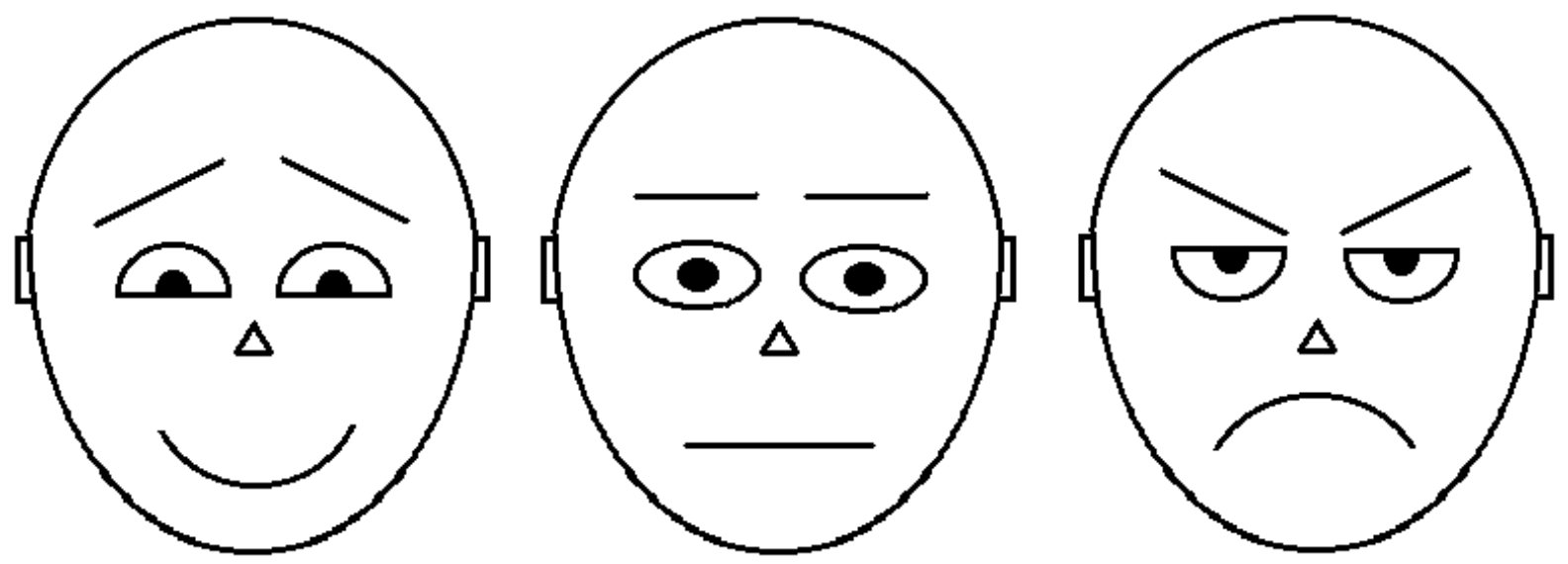

Figure 1. Schematic representation of happy, neutral and threatening human faces (adapted from Öhman, Lundqvist \& Esteves, 2001) 

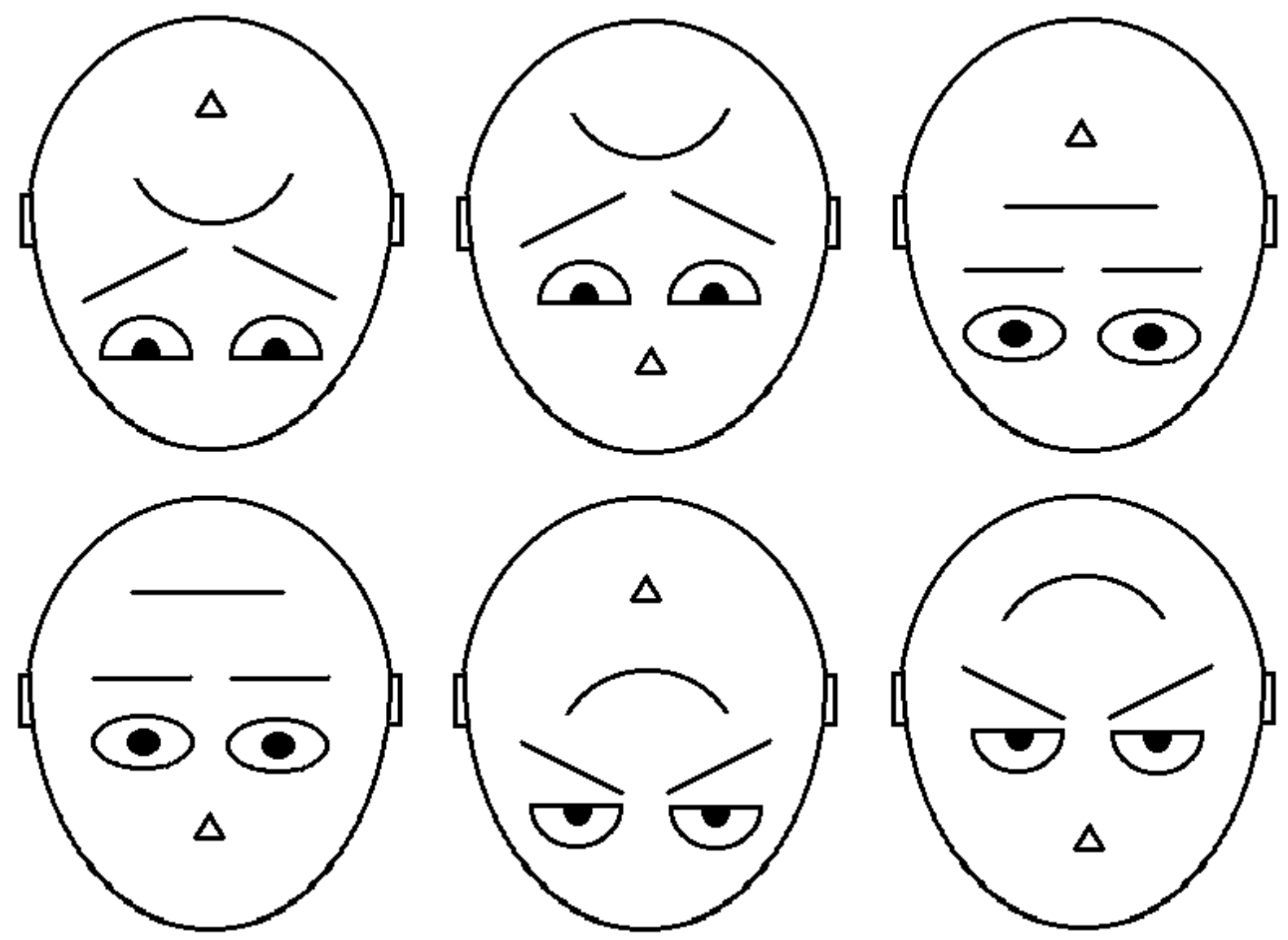

Figure 2. The six scrambled faces that served as stimuli for the threshold setting and final awareness checking trials
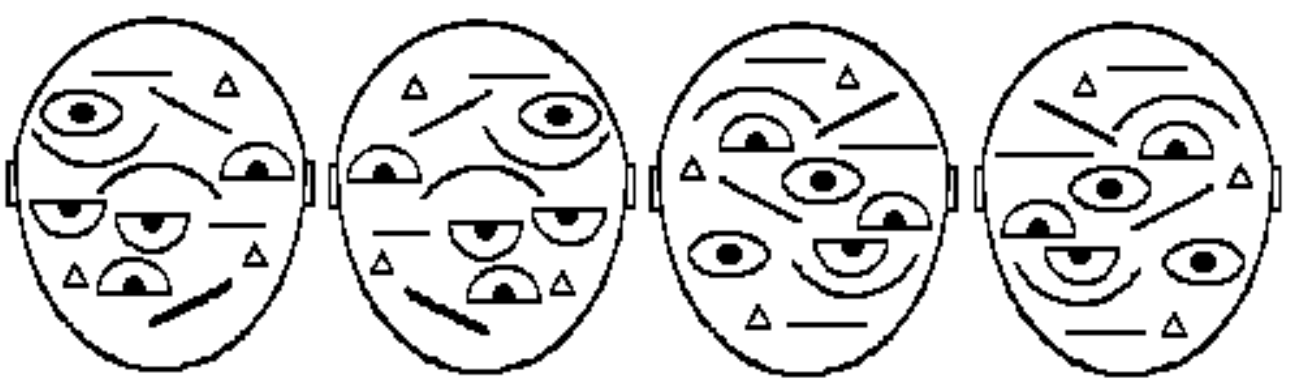

Figure 3. The four pattern masks

Probe stimuli. Two geometric shapes (circle or square) were used as the probes. The probes were presented in dimensions approximately $2.5 \mathrm{~mm}$ wide in solid black colouring approximately $100 \mathrm{~mm}$ to either side of the central fixation point.

Arousal Rating Questionnaire. The Arousal Rating Questionnaire is a brief, self-report measure of fearfulness, nervousness and anxiousness. Scores on the measure are positively associated with scores on the STAI-S (see Edwards et al., 2006). The statement "Right now, at this moment I feel" preceded the dimensions of nervous to calm, fearful to not-at-all fearful, and anxious to not-at-all anxious. Participants responded to each dimension on a seven-point scale with the range 3-2-1-0-1-2-3. For each dimension a score of 3 reflected a rating of very for that dimension (e.g., nervous or calm) a score of 2 quite, a score of 1 slightly, and a rating of zero indicated that the participant felt neither one end of the scale or the other. 


\subsection{Apparatus}

Experimental hardware. The stimuli were presented by a Dell OptiPlex GX110 Pentium 3 computer running at $866 \mathrm{MHz}$ using a Video Stimulus Generator video card (VSG; 2-3 issue 4a) capable of refresh rates up to 500 $\mathrm{Hz}(2 \mathrm{~ms})$. Stimuli were presented on a Hitachi Superscan 81321 -inch colour monitor with a vertical refresh rate of $200 \mathrm{~Hz}(5 \mathrm{~ms})$. A custom-built two-button response box labelled ODD (left button) and EVEN (right button) was attached to the computer and used for the digit classification task. The box was labelled FACE (left button) and NON-FACE (right button) for the awareness check trials. Probe-classification responses were detected by headset microphone connected to the computer.

Experimental software. The VSG software controlled the presentation of stimuli for the practice trials, digit classification task, probe-classification tasks, and awareness check trials. The software also recorded reaction latencies and errors.

Electric stimulus. A Grass SD9 stimulator $(0-90 \mathrm{~V})$ delivered the $200 \mathrm{~ms}$ electric stimulus through a $35 \mathrm{~mm}$ diameter concentric stainless steel electrode. Electrode-skin contact was made through a sponge soaked in saline.

\subsection{Design}

A 2 X 3 X 2 X 2 mixed factorial design was used. The between groups factor was Trait Anxiety (high vs. low), whereas the within groups factors were Valence (happy, neutral, threat), Exposure Mode (masked vs. unmasked), and Shock Condition (shock threat vs. shock safe). The dependent variable was probe-classification latency.

\subsection{Procedure}

Participants were tested individually and testing took approximately 40 minutes each. After informed consent was obtained, participants completed the STAI-State, STAI-Trait, BDI-IA, MCSDS, and the Arousal Rating Questionnaire. They then undertook the shock setting procedure, the reaction time trials, and a series of awareness checking trials.

Shock intensity setting. The intensity of the electric stimulus was determined individually. The electrode was attached to the fleshy surface of the participant's forearm and starting from a baseline of $0 \mathrm{~V}$ the shocks were presented in $10 \mathrm{~V}$ increments until the participant reported the intensity to be "unpleasant, but not painful." Following this procedure the shock electrode was removed.

Experimental trials. There were 4 blocks of 24 experimental trials. Each trial involved two tasks: (1) a digit classification task, and (2) a probe classification task. Participants were instructed to classify the status of both the digit and probe as quickly as possible without making too many mistakes, and to ignore all other information that was presented during the trials. On each trial, a fixation cue of three white crosses was presented for $1 \mathrm{~s}$ in the centre of the computer screen. The screen was then blanked for $250 \mathrm{~ms}$ after which a digit between 2 and 9 was presented in the location formerly occupied by the cue. Digits were presented in a character approximately $10 \mathrm{~mm}$ high. On half of the trials the status of the digit was odd whereas for the other half it was even. Digit status was quasi-randomised such that not more than 4 trials of the same status were presented in succession. Digit classification was made using a button box. The digit remained on the screen until the participant's response was detected by the software at which time the screen was blanked. Immediately following the offset of the digit, one of the geometric probes and one of the schematic faces were presented concurrently. Probes were presented approximately $100 \mathrm{~mm}$ either to the left or right of the fixation point, whereas the faces appeared above and below the central fixation point. The centre-to-centre separation of the faces was approximately 70 $\mathrm{mm}$. The participant's second task was to verbally classify the shape of the probe as quickly as possible. Once the software detected the response the screen was blanked. On half of the trials the distracting faces were presented masked and the other half they were unmasked. Exposure mode was randomly intermixed but governed by the parameter that not more than two trials of the same exposure mode occurred in succession.

Within each block, the three face stimuli were presented on eight occasions; four trials for each face type were presented masked and four were unmasked. The combination of pattern masks and face types was balanced such that each combination was presented equally often across the first and second two blocks of trials. Face type and exposure mode were randomised but the order of presentation was restricted such that not more than two trials of the same face or exposure mode occurred in succession. Probes appeared with equal probability and in either location with equal probability. Probe type and probe position was balanced with face valence and exposure mode within blocks. Probe type and position were quasi-randomised such that not more than two trials of the same type occurred in succession.

On unmasked trials the face information remained on the screen until the software detected the participant's first vocal response to the status of the probe. For the masked trials the latency between the target item and the mask 
was set at $15 \mathrm{~ms}$. On these trials, once the mask replaced the target, it remained on the screen until the software detected the participant's response at which time the screen was blanked. Experimenter coding of responses initiated the next trial and the inter-trial interval was approximately $4 \mathrm{~s}$.

Prior to undertaking the experimental trials, participants completed a block of 24 practice trials for familiarisation purposes. The parameters for the practice trails were identical to those used for the main task with the exception that the scrambled faces were used as the stimuli. For the practice trials, the combination of faces, masks, probe types and probe locations was fully balanced. Following the practice trials, and prior to undertaking the main task, participants were allowed a three-minute rest. During this period, half had the shock electrode re-attached to their forearm (shock threat group). Assignment to the initial shock threat and shock safe groups was based on order of arrival at the laboratory and trait anxiety status. The shock threat group were informed that the software would deliver between 3 and 5 shocks at random over the first two blocks of trials (in reality only two shocks were delivered), and that the delivery of shocks was not contingent on performance. Those in the shock threat group received one shock approximately $15 \mathrm{~s}$ prior to the first trial and immediately following the final trail in the first block. They were informed it was possible that shocks could be delivered during the rest period between blocks, and to confirm the efficacy of the threat of shock in elevating state anxiety, participants completed the arousal rating questionnaire during the break between blocks. Following the second block of trials the shock threat and shock safe groups were reversed. The second shock threat group received a shock approximately $15 \mathrm{~s}$ prior to the first trial and immediately following the final trial in the third block. This group was also warned that they might receive further shocks during the rest period between the blocks and once they had indicated that this instruction was understood they completed the Arousal Rating Questionnaire. Following completion of the fourth block of trials this group had the electrode removed.

Awareness check trials. To confirm that participants were unaware of the nature of the items on masked trials a block of 48 awareness check trials was completed. These trials were similar to the experimental trials with the exception that the task was to verbally classify the status of the target faces (i.e., face vs. non-face) rather than the status of the probe. To ensure that participants could discriminate between the faces and non-faces, they were shown printed versions of the items. Each trial involved the digit classification task, after which either a face or non-face pair was presented for $15 \mathrm{~ms}$ and immediately masked. The spatial location of the face stimuli was identical to the experimental trials. Half the trials were faces and half were non-faces, and participants were encouraged to guess when unsure. The order of presentation was quasi-randomised such that not more than two items of the same status occurred in succession. The data of participants who made 27 or fewer correct identifications were retained for further analyses. Following these trials participants were debriefed, thanked and released.

\section{Results}

\subsection{Manipulation Checks}

Validity of trait anxiety status. A between groups $t$ test confirmed that the HTA group reported significantly higher trait anxiety $(M=45.41 ; S D=7.19)$ than the LTA group $(M=30.22 ; S D=4.42), t(62)=10.18, p<.001$. The HTA group also reported higher state anxiety $(M=39.53 ; S D=7.98), t(62)=5.70, p<.001$, and depression, $(M=9.16 ; S D=3.71), t(62)=5.44, p<.001$, than the LTA group $(M \mathrm{~s}=29.13$ and $4.56 ; S D \mathrm{~s}=6.57$ and 2.97$)$. The groups were matched on social desirability, $t(62)=1.58, p=1.20$, n.s.

Validity of state anxiety manipulation. Mean shock intensity for the HTA (40.47 V) and LTA (41.72 V) groups did not vary, $t<1$. The mean ratings on the arousal-rating questionnaire were calculated for the HTA and LTA groups in the shock threat and shock safe conditions, and these data are shown in Table 1. Repeated measures $t$-tests on the arousal rating data for the HTA and LTA groups performing with and without the threat of shock confirmed higher state anxiety in both groups and on all dimensions when under the threat of shock, all $t(31)>$ 4.97, all $p<.001$. 
Table 1. Means and standard deviations of responses for HTA and LTA participants on arousal rating questionnaire dimensions under shock threat and shock safe conditions

\begin{tabular}{|c|c|c|c|c|c|c|c|c|}
\hline \multirow[b]{3}{*}{ Variable } & \multicolumn{4}{|c|}{ High Trait Anxious } & \multicolumn{4}{|c|}{ Low Trait Anxious } \\
\hline & \multicolumn{2}{|c|}{ Shock Safe } & \multicolumn{2}{|c|}{ Shock Threat } & \multicolumn{2}{|c|}{ Shock Safe } & \multicolumn{2}{|c|}{ Shock Threat } \\
\hline & $\mathrm{M}$ & SD & $\mathrm{M}$ & SD & $\mathrm{M}$ & SD & $\mathrm{M}$ & SD \\
\hline Nervous-Calm & -0.25 & 1.37 & 1.41 & 1.13 & -1.50 & 1.91 & -0.34 & 1.66 \\
\hline Fearful-Not Fearful & -0.97 & 1.20 & 0.78 & 1.54 & -2.00 & 1.08 & -0.16 & 1.61 \\
\hline Anxious-Not Anxious & -0.00 & 1.41 & 1.56 & 1.24 & -1.72 & 1.30 & -0.50 & 1.69 \\
\hline
\end{tabular}

Note. Positive scores denote greater nervousness, fearfulness and anxiety whereas negative scores denote the opposite.

Validity of masking procedure in preventing awareness. The percentage of correct face status decisions on the final awareness checking trials did not differ between the HTA (47.06\%) and LTA (47.39\%) groups, $t<1$. The overall mean accuracy of both groups was 22.67 out of $48(S D=3.16)$, which did not differ from that expected by chance, $z=.42$, n.s. These data suggest that it was unlikely participants were aware of the face status of the items during the masked trials.

\subsection{Data Reduction}

Prior to analysis the data were reduced in four stages: (a) microphone failures $(0.41 \%$ of trials), (b) probe classification errors (3.19\% of trials), (c) response times less than $300 \mathrm{~ms}$ or greater than $3000 \mathrm{~ms}(0.27 \%$ of trials), and (d) trials more than 2 standard deviations from each participant's cell mean ( $2.91 \%$ of trials) were removed.

\subsection{Reaction Time Data}

Mean response latencies in each experimental cell were extracted for each participant. The means and standard deviations for the groups in each experimental cell are shown in Table 2. The reaction time data were analysed in a 3 X 2 X 2 X 2 mixed design factorial ANOVA with Valence (happy, neutral, threat faces), Exposure Mode (masked vs. unmasked), and Shock Condition (shock threat vs. shock safe) as within groups factors, and Trait Anxiety (high vs. low) as a between groups factor.

Table 2. Means and standard deviations of probe detection latency data in milliseconds for high trait anxious and low trait anxious participants in each experimental condition

\begin{tabular}{|c|c|c|c|c|c|c|c|c|}
\hline \multirow[b]{3}{*}{ Variable } & \multicolumn{4}{|c|}{ High Trait Anxious } & \multicolumn{4}{|c|}{ Low Trait Anxious } \\
\hline & \multicolumn{2}{|c|}{ Shock Safe } & \multicolumn{2}{|c|}{ Shock Threat } & \multicolumn{2}{|c|}{ Shock Safe } & \multicolumn{2}{|c|}{ Shock Threa } \\
\hline & $\mathrm{M}$ & SD & $\mathrm{M}$ & SD & $\mathrm{M}$ & SD & $\mathrm{M}$ & SD \\
\hline \multicolumn{9}{|l|}{ Masked } \\
\hline Happy & 707 & 80 & 716 & 73 & 686 & 81 & 668 & 62 \\
\hline Neutral & 698 & 65 & 705 & 77 & 674 & 73 & 690 & 70 \\
\hline Threat & 704 & 78 & 703 & 68 & 667 & 66 & 688 & 81 \\
\hline \multicolumn{9}{|c|}{ Unmasked } \\
\hline Happy & 696 & 86 & 700 & 72 & 671 & 68 & 676 & 68 \\
\hline Neutral & 699 & 77 & 703 & 72 & 672 & 77 & 677 & 68 \\
\hline Threat & 692 & 78 & 717 & 80 & 676 & 86 & 670 & 82 \\
\hline
\end{tabular}

The results yielded a marginally significant effect for Exposure Mode, $F(1,62)=3.87, M S E=1155.82, p=.054$, $\eta^{2}=.06$, with a tendency for longer response latencies on masked trials $(M=692 \mathrm{~ms})$ than on unmasked trials $(M=687 \mathrm{~ms})$. The only other significant effect to emerge was the interaction involving all four factors, $F(2,124)$ 
$=5.17, M S E=1201.42, p=.007, \eta^{2}=.08$. This finding suggested that the probe identification latencies varied between the HTA and LTA groups for the masked and unmasked trials, and that these latencies were further influenced by both shock condition and valence of the items. The pattern of the interaction is represented in Figure 4. To facilitate understanding of how the probe classification data was influenced by the experimental manipulations, separate analyses were preformed for the masked and unmasked trials.

Masked trials. The only significant effect to emerge from analyses performed on the masked trials was a Trait Anxiety X Shock Condition X Valence interaction, $F(2,124)=4.56, M S E=1127.50, p=.012, \eta^{2}=.07$. The top panels of Figure 4 summarise the nature of this interaction. As the top left panel of the figure shows, for the HTA group neither item valence or shock condition status affected response latency to the probes on the masked trials, all $F<1.59, p>.212$, n.s. As can be seen in the top right panel of the figure however, the pattern of responding was not equivalent for the LTA group. This observation was confirmed by a significant Shock Condition X Valence interaction, $F(2,62)=5.46, M S E=1369.08, p=.007, \eta^{2}=.15$. Within subjects $t$ tests revealed that in the shock safe condition, response latencies were significantly faster on threat trials than on happy trials, $t(31)=$ $2.48, p=.019$, with reaction times on neutral trials being not statistically different from either, both $t<1.22$, $p>.23$, n.s. In the shock threat condition however, this pattern was almost reversed. That is, LTA participants demonstrated significantly longer probe reaction latencies during both threat trials, $t(31)=2.34, p=.026$, and neutral trials, $t(31)=2.82, p=.008$, than during happy trials. Response latencies on threat and neutral trials did not differ, $t<1$.

Unmasked trials. The data for the unmasked trials are shown in the bottom panels of Figure 4. When an equivalent set of analyses were performed for the unmasked trials the results failed to produce any significant main effects or interactions, all $F<2.64, p>.109$, n.s. 

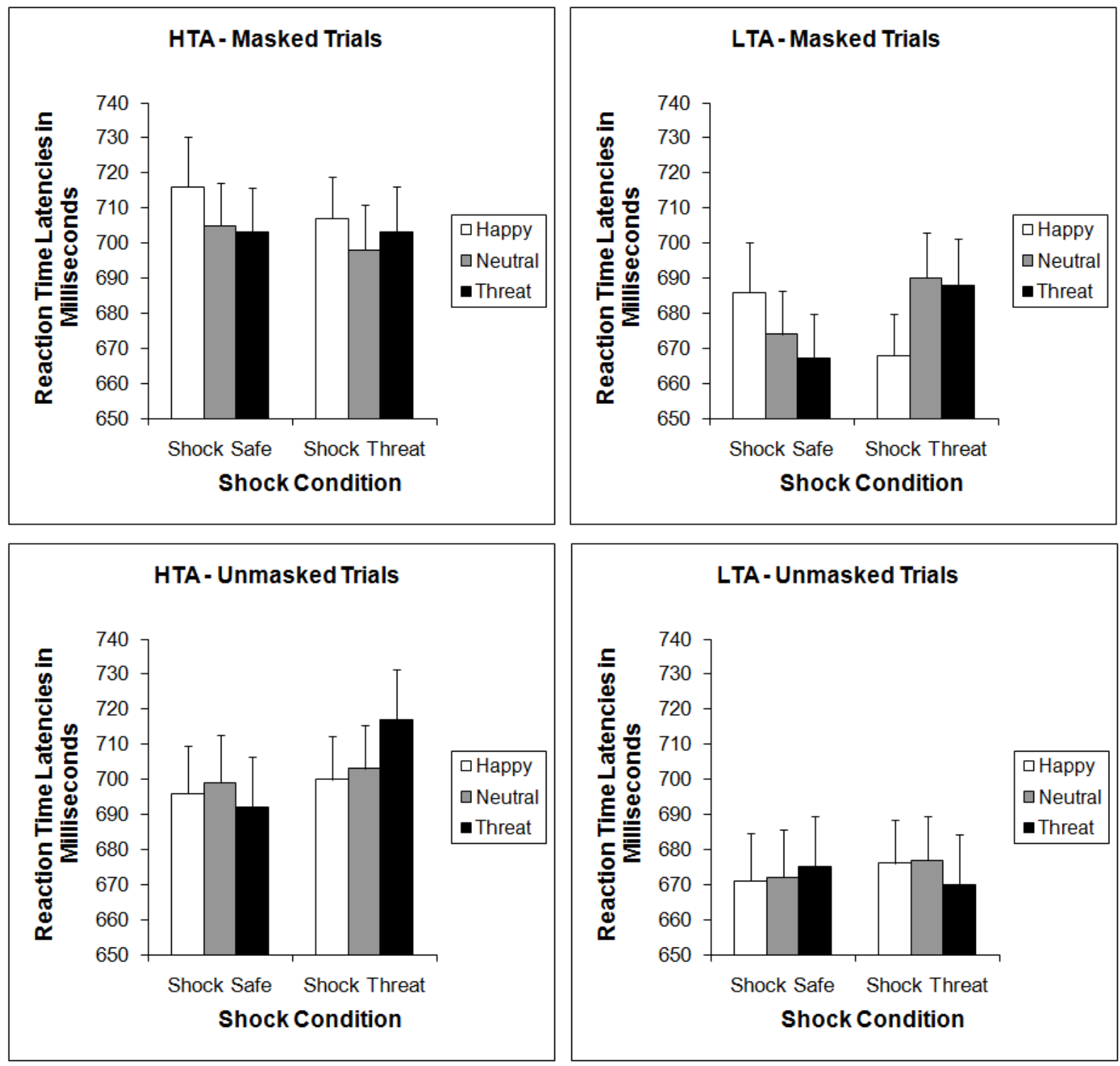

Figure 4. Mean probe reaction time latencies in milliseconds for the HTA and LTA groups in the shock threat and shock safe conditions as a function of item valence and exposure mode (vertical bars represent standard errors of the mean)

\subsection{Depression Analyses}

Given that the self-reported trait anxiety and depression scores were related, a parallel set of analyses to that performed on the trait anxiety data were undertaken after reassigning participants to high and low depression groups based on a median split of BDI-IA scores. These analyses failed to produce any significant main effects or interactions involving Depression Group, all $F<1.85, p>.162$, n.s.

\section{Discussion}

The present experiment was designed to investigate whether selective attention for differentially valenced schematic faces proceeds without volition and awareness, and to evaluate the effects of state and trait anxiety on processing with respect to the predictions made by the theories of Bower (1981), Mogg and Bradley (1998), and Williams et al. (1988, 1997). A novel interference methodology was developed to overcome limitations associated with the emotional Stroop and dot-probe tasks by presenting the threat and control items in a location not focal to the central task. Disruption to probe-classification latencies was taken as an index of the degree to which the threatening and non-threatening materials had selectively recruited attention. Bower's semantic network model predicts that participants under high stress conditions would be slower to identify the status of 
probes on threat trials relative to control trials, irrespective of trait anxiety status. The models of Williams et al. and Mogg and Bradley, however, predict that state and trait anxiety combine to moderate selective attention. Specifically, the Williams et al. model suggests that HTA individuals under a high stress condition would be slower to identify the status of the probe on threat faces trials, whereas LTA participants would demonstrate a threat avoidance effect, as evidenced by faster probe classification latencies on threat face trials. The Mogg and Bradley model, however, predicts that both HTA and LTA individuals performing under the threat of shock would be disrupted on the probe classification task on threat face trials relative to control trials. The probe classification data for the masked trials suggested that LTA participants were selectively avoidant of threat without the threat of shock, and attended to threat under the threat of shock. There was no evidence for pre-conscious selective threat processing in HTA participants, and no evidence of selective processing in either group when post-conscious access to the material was permitted.

During masked trials, LTA participants in the shock safe condition were significantly slower to classify the status of probes during happy face trials, compared to threat face trials. During the high state anxiety condition, however, this pattern was reversed. When performing under the threat of shock, LTA individuals were significantly slower at identifying the status of the probe on both threat and neutral trials, relative to happy trials. These data therefore suggest that LTA individuals were vigilant for positively valenced material when state anxiety was low, whereas under high stress conditions they selectively attend to potentially threatening faces relative to the happy faces. It is important to note that in the shock condition there was no difference in response latency between the threat and neutral items. Given that neutral faces might sometimes be interpreted as being slightly hostile, attending to them under conditions of restricted awareness during periods of elevated state anxiety may be less costly in terms of survival than choosing to ignore them (cf. Öhman \& Mineka, 2001). The results for the LTA individuals during the masked trials seem consistent with the framework of Bower (1981) which predicts that selective attention effects are moderated by current arousal. However, whereas Bower's model predicts that this pattern of responding should be observed for all individuals, in the present study it was restricted to the LTA group.

The results for the LTA participants during the masked trials were not consistent with the theorising of Williams et al. $(1988,1997)$. According to their model, as threat value and/or state anxiety increases LTA participants should become more avoidant of threat. In the present study it seemed that LTA individuals were vigilant for happy faces during periods of low state arousal, however, under higher state anxious conditions it seems that their attentional system switches to a mode that is vigilant for threat. The data did, however, offer partial support for the Mogg and Bradley (1998) model. Their framework predicts that irrespective of trait anxiety status, all individuals should become more vigilant for higher threat value stimuli, which seemed to be the case here. Nonetheless, it should be noted that the Mogg and Bradley's framework is not specific with respect to the relative influence of threat value and/or state anxiety in producing this pattern of attentional responding. The observation that the LTA group was vigilant for threat only during the shock threat trials suggests that state anxiety plays a critical role in initiating this pattern of responses.

For HTA individuals there was no evidence for selective processing of threat during the masked exposure trials in either the shock safe or shock threat condition. On the basis of the data reported here, it would appear that HTA individuals attend selectively to briefly presented pictorial threat material when it is presented in attended-to locations (e.g., Mogg \& Bradley, 1999), but when differentially valenced faces are presented in task-irrelevant locations, it seems that they do not apportion attention on the basis of the nature of the content. If it is adaptive to be vigilant for briefly presented representations of biologically significant threat appearing in non-attended-to locations when aroused (as observed in the LTA group), then the attentional patterns of HTA individuals might not so much be characterised as being maladaptive, but more so by a pattern that suggests they do not initiate adaptive responses.

The data observed for the unmasked trials were discordant to those obtained for the masked trials. For both the HTA and LTA groups, response latencies on the probe classification task were unaffected by valence or shock condition. Unlike the results for the masked exposure trials, the LTA individuals showed no evidence of a mood congruent processing bias when they had conscious access to the stimulus material. It therefore appears that when awareness of the stimuli is permitted, the presence of distracting differentially valenced pictorial information does not affect the ability of LTA individuals to perform task relevant demands (cf. Williams et al., 1997). Importantly, it also seems that for LTA individuals this ability is unaffected by the nature of task (i.e., emotional Stroop, dot-probe, current task) or by whether the distracting information is presented in attended (e.g., Mogg \& Bradley 1999) or non-attended locations (current study). 
The pattern of responses during the unmasked exposure trials does not support the Bower (1981), Mogg and Bradley (1998) and Williams et al. $(1988,1997)$ models. For example, according to the Williams et al. framework, as threat value increases LTA individuals should display increased attentional avoidance of threat, whereas HTA individuals should become more vigilant for threat. Mogg and Bradley, on the other hand, predict that higher intensity threat material should produce vigilance for threat in both HTA and LTA individuals. Previous studies using dot-probe procedures have found that all individuals respond to the location of probes replacing pictures of threatening facial expressions faster than to probes replacing happy (e.g., Mogg \& Bradley, 1999) and neutral facial expressions (e.g., Wilson \& MacLeod, 2003). If it is accepted that representations of threatening human faces represent a greater threat value than verbal material, then the findings of these earlier studies are in agreement with the direction of the bias proposed by Mogg and Bradley. Nonetheless, the data reported in these previous studies are constrained by the limitations associated with previous versions of the dot-probe task.

The data reported in the present experiment for the unmasked trials are, however, consistent with those reported by van Honk et al. (2001) who also employed an interference paradigm and failed to find selective threat effects under conditions permitting conscious access to the material. Considered together, these finding suggest that both HTA and LTA individuals might attend to the spatial location of threatening facial stimuli at the post-conscious level, but that the effect is restricted to conditions in which the information is presented in task-relevant locations and under conditions in which there is limited competition for attentional resources.

A possible limitation of the present experiment concerns the schematic stimuli employed. Because the stimuli were not photographic representations of actual faces it is not possible to draw firm conclusions about the nature of selective attention effects for real world facial threat. Nonetheless, the use of schematic facial stimuli represents a considerable improvement over the use of verbal material, and overcomes a number of the procedural and interpretational problems associated with the use of photographic pictures of actual faces (see Öhman et al., 2001). Careful control was exercised over line thickness, luminance, and the specific features representing each emotional state, and therefore the items were considered the most appropriate option to test the hypotheses under investigation. A second cautionary note concerns the fact that awareness checks for the masked trials were carried out using a face/non-face discrimination task, rather than a discrimination task based on emotional expression. This procedure raises the possibility that participants might have been aware of the facial emotions during the masked trials and brings to question the efficacy of the exposure manipulation. Nonetheless, the fact response times to the differentially valenced items varied as a function of exposure mode (and the anxiety factors as evidenced in the 4-way interaction) suggests that the manipulation was successful. More importantly, chance performance on a face/non-face discrimination task would subsume a discrimination task based on emotional expression; if participants were unable to determine whether a face was presented then it would be unlikely they could determine its emotional tone.

In summary, the data reported in the present experiment suggest that selective attention for schematic representations of differentially valenced human faces is influenced by state anxiety status, trait anxiety status, and by the level of participants' awareness when the to-be-attended and to-be-ignored information is presented in spatially separate locations. For the masked trials, LTA individuals demonstrated an adaptive mood congruent attentional bias. Vigilance for positive material might serve as a mood regulation mechanism when state anxiety is low (cf. MacLeod \& Rutherford, 1992), whereas vigilance for briefly presented biologically relevant threat when state anxiety is high would facilitate initiation of a response to potential danger (cf. Öhman \& Mineka, 2001). For the HTA participants, there was no evidence for selective attention during the masked exposures. If it is considered adaptive to initiate a preconscious attentional response to biologically relevant threat, then the data suggests that anxiety might be characterised by a failure to respond appropriately. During the unmasked trials, there was no evidence of an attentional bias as a function of trait or state anxiety status. This finding suggests that participants are not distracted by emotionally valenced information of which they are aware, and raises questions as to whether biases for pictorial threat operate without volition at the post-conscious level under conditions that require competition for attentional resources.

\section{References}

Amir, N., Freshman, M., \& Foa, E. B. (2002). Enhanced Stroop interference for threat in social phobia. Journal of Anxiety Disorders, 16, 1-9. http://dx.doi.org/10.1016/S0887-6185(01)00084-6

Asmundson, G. J. G., \& Stein, M. B. (1994). Selective processing of social threat in patients with generalized social phobia: Evaluation using a dot-probe paradigm. Journal of Anxiety Disorders, 8, 107-117. http://dx.doi.org/10.1016/0887-6185(94)90009-4 
Beck, A. T., \& Steer, R. A. (1993). Manual for the Beck Depression Inventory. San Antonio, TX: Psychological Corporation.

Bower, G. (1981). Mood and memory. American Psychologist, 36, 129-148. http://dx.doi.org/10.1037/0003-066X.36.2.129

Bradley, B. P., Mogg, K., Falla, S. J., \& Hamilton, L. R. (1998). Attentional bias for threatening facial expressions in anxiety: Manipulation of stimulus duration. Cognition and Emotion, 12, 737-753. http://dx.doi.org/10.1080/026999398379411

Bradley, B. P., Mogg, K., Millar, N., \& White, J. (1995). Selective processing of negative information: Effects of clinical anxiety, concurrent depression, and awareness. Journal of Abnormal Psychology, 104, 532-536. http://dx.doi.org/10.1037/0021-843X.104.3.532

Broadbent, D., \& Broadbent, M. (1988). Anxiety and attentional bias: State and trait. Cognition and Emotion, 2, 165-183. http://dx.doi.org/10.1080/02699938808410922

Cohen, Y., Lachenmeyer, J. R., \& Springer, C. (2003). Anxiety and selective attention in obsessive-compulsive disorder. Behaviour Research and Therapy, 41, 1311-1323. http://dx.doi.org/10.1016/S0005-7967(03)00037-8

Crowne, D. P., \& Marlowe, D. (1960). A new scale of social desirability independent of psychopathology. Journal of Consulting Psychology, 24, 349-354. http://dx.doi.org/10.1037/h0047358

Edwards, M. S., Burt, J. S., \& Lipp, O. V. (2006). Selective processing of masked and unmasked verbal threat material in anxiety: Influence of an immediate acute stressor. Cognition \& Emotion, 20, 812-835. http://dx.doi.org/10.1080/02699930500375761

Edwards, M. S., Burt, J. S., \& Lipp, O. V. (2010a). Selective attention for masked and unmasked emotionally toned stimulus information: Effects of trait anxiety, state anxiety and test order. British Journal of Psychology, 101, 325-343. http://dx.doi.org/10.1348/000712609X466559

Edwards, M. S., Burt, J. S., \& Lipp, O. V. (2010b). Selective attention for masked and unmasked threatening words in anxiety: Effects of trait anxiety, state anxiety and awareness. Behaviour Research and Therapy, 48, 210-218. http://dx.doi.org/10.1016/j.brat.2009.11.003

Foa, E. B., Feske, U., Murdock, T. B., Kozak, M. J., \& McCarthy, P. R. (1991). Processing of threat related information in rape victims. Journal of Abnormal Psychology, 100, 156-162. http://dx.doi.org/10.1037/0021-843X.100.2.156

Fox, E. (1996). Selective processing of threat words in anxiety: The role of awareness. Cognition and Emotion, 10, 449-480. http://dx.doi.org/10.1080/026999396380114

Harvey, A. G., Bryant, R. A., \& Rapee, R. M. (1996). Preconscious processing of threat in posttraumatic stress disorder. Cognitive Therapy and Research, 20, 613-623. http://dx.doi.org/10.1007/BF02227964

Hunt, C., Keogh, E., \& French C. C. (2006). Anxiety sensitivity: The role of conscious awareness and selective attentional bias to physical threat. Emotion, 6, 418-428. http://dx.doi.org/10.1037/1528-3542.6.3.418

Koster, E. H. W., Verschuere, B., Burssens, B., Custers., R., \& Crobmez, G. (2007). Attention for emotional faces under restricted awareness revisited: Do emotional faces automatically attract attention? Emotion, 7 , 285-295. http://dx.doi.org/10.1037/1528-3542.7.2.285

Lundh, L. -G., Wikström, J., Westerlund, J., \& Öst, L. -G. (1999) Preattentive bias for emotional information in panic disorder with agoraphobia. Journal of Abnormal Psychology, 108, 222-232. http://dx.doi.org/10.1037/0021-843X.108.2.222

Lundqvist, D., Esteves, F., \& Öhman, A. (1999). The face of wrath: Critical features for conveying facial threat. Cognition and Emotion, 13, 691-711. http://dx.doi.org/10.1080/026999399379041

MacLeod, C., \& Rutherford, E. M. (1992). Anxiety and the selective processing of emotional information: mediating roles of awareness, state and trait variables, and personal relevance of stimulus materials. Behaviour Research and Therapy, 30, 479-491. http://dx.doi.org/10.1016/0005-7967(92)90032-C

MacLeod, C., \& Mathews, A. (1988). Anxiety and the allocation of attention to threat. The Quarterly Journal of Experimental Psychology, 40, 653-670. http://dx.doi.org/10.1080/14640748808402292

MacLeod, C., Mathews, A., \& Tata, P. (1986). Attentional bias in emotional disorders. Journal of Abnormal Psychology, 95, 15-20. http://dx.doi.org/10.1037/0021-843X.95.1.15 
McNally, R. J., Riemann, B. C., \& Kim, E. (1990). Selective processing of threat cues in panic disorder. Behaviour Research and Therapy, 28, 407-412. http://dx.doi.org/10.1016/0005-7967(90)90160-K

Miller, M. W., \& Patrick, C. J. (2000). Trait differences in affective and attentional responding to threat revealed by emotional Stroop interference and startle reflex modulation. Behavior Therapy, 31, 757-776. http://dx.doi.org/10.1016/S0005-7894(00)80043-X

Mogg, K., \& Bradley, B. P. (1998). A cognitive-motivational analysis of anxiety. Behaviour Research and Therapy, 36, 809-848. http://dx.doi.org/10.1016/S0005-7967(98)00063-1

Mogg, K., \& Bradley, B. P. (1999). Orienting of attention to threatening facial expressions presented under conditions of restricted awareness. Cognition and Emotion, 13, 713-740. http://dx.doi.org/10.1080/026999399379050

Mogg, K., Bradley, B. P., \& Hallowell, N. (1994). Attentional bias to threat: Roles of trait anxiety, stressful events, and awareness. The Quarterly Journal of Experimental Psychology, 47A, 841-864. http://dx.doi.org/10.1080/14640749408401099

Mogg, K., Bradley, B. P., \& Williams, R. (1995). Attentional bias in anxiety and depression: The role of awareness. British Journal of Clinical Psychology, 34, 17-36. http://dx.doi.org/10.1111/j.2044-8260.1995.tb01434.x

Mogg, K., Mathews, A., Bird, C., \& Macgregor-Morris, R. (1990). Effects of stress and anxiety on the processing of threat stimuli. Journal of Personality and Social Psychology, 59, 1230-1237. http://dx.doi.org/10.1037/0022-3514.59.6.1230

Mogg, K., Mathews, A., \& Eysenck, M. (1992). Attentional bias to threat on clinical anxiety states. Cognition and Emotion, 6, 149-159. http://dx.doi.org/10.1080/02699939208411064

Mogg, K., McNamara, J., Powys, M., Rawlinson, H., Seiffer, A., \& Bradley, B. P. (2000). Selective attention to threat: A test of two cognitive models of anxiety. Cognition and Emotion, 14, 375-399. http://dx.doi.org/10.1080/026999300378888

Öhman, A, Lundqvist, D., \& Esteves, F. (2001). A face in the crowd revisited: A threat advantage with schematic stimuli. Journal of Personality and Social Psychology, 80, 381-396. http://dx.doi.org/10.1037/0022-3514.80.3.381

Öhman, A., \& Mineka, S. (2001). Fears, phobias, and preparedness: Toward an evolved module of fear and fear learning. Psychological Review, 108, 483-522. http://dx.doi.org/10.1037/0033-295X.108.3.483

Rutherford, E. M., MacLeod, C., \& Campbell, L. W. (2004). Negative selectivity effects and emotional selectivity effects in anxiety: Differential attentional correlates of state and trait variables. Cognition and Emotion, 18, 711-720. http://dx.doi.org/10.1080/02699930341000121

Schmukle, S. C. (2005). Unreliability of the dot probe task. European Journal of Personality, 19, 595-605. http://dx.doi.org/10.1002/per.554

Spielberger, C. D., Gorsuch, R. L., Lushene, R. E., Vagg, P. R., \& Jacobs, G. A. (1983). Manual for the State-Trait Anxiety Inventory (Form Y). Palo Alto CA: Consulting Psychologists Press.

Strahan, R., \& Gerbasi, K. C. (1972). Short, homogeneous versions of the Marlowe-Crown social desirability

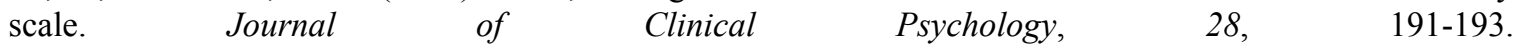
http://dx.doi.org/10.1002/1097-4679(197204)28:2<191::AID-JCLP2270280220>3.0.CO;2-G

Tata, P., Leibowitz, J. A., Prunty, M. J., Cameron, M., \& Pickering, A. D. (1996). Attentional bias in obsessional compulsive disorder. Behaviour Research and Therapy, 34, 53-60. http://dx.doi.org/10.1016/0005-7967(95)00041-U

Thorpe, S. J., \& Salkovskis, P. M. (1997). Information processing in spider phobics: The Stroop colour naming task may indicate strategic but not automatic attentional bias. Behaviour Research \& Therapy, 35, 131-144. http://dx.doi.org/10.1016/S0005-7967(96)00093-9

van Honk, J., Tuiten, A., de Haan, E., van den Hout, M., \& Stam, H., (2001). Attentional biases for angry faces: Relationship to trait anger and anxiety. Cognition and Emotion, 15, 279-297. http://dx.doi.org/10.1080/02699930126112 
Weinberger, D. A., Schwartz, G. E., \& Davidson, J. R. (1979). Low-anxious, high-anxious, and repressive coping styles: Psychometric patterns and behavioural and physiological responses to stress. Journal of Abnormal Psychology, 88, 369-380. http://dx.doi.org/10.1037/0021-843X.88.4.369

Williams, J. M. G., Watts, F. N., MacLeod, C., \& Mathews, A. (1988). Cognitive psychology and emotional disorders. Chichester England: Wiley.

Williams, J. M. G., Watts, F. N., MacLeod, C., \& Mathews, A. (1997). Cognitive psychology and emotional disorders (2nd ed.). Chichester England: Wiley.

Wilson, E., \& MacLeod, C. (2003). Contrasting two accounts of anxiety-linked attentional bias: Selective attention to varying levels of stimulus threat intensity. Journal of Abnormal Psychology, 112, 212-218. http://dx.doi.org/10.1037/0021-843X.112.2.212

\section{Copyrights}

Copyright for this article is retained by the author(s), with first publication rights granted to the journal.

This is an open-access article distributed under the terms and conditions of the Creative Commons Attribution license (http://creativecommons.org/licenses/by/3.0/). 